\title{
Characterization study of polyAMPS@BMA core-shell particles using two types of RAFT agents
}

\author{
Nasrullah Shah ${ }^{1, *}$, Zubair Ullah Khan ${ }^{1}$, Manzoor Hussain ${ }^{1}$, Touseef Rehan ${ }^{2}$, Abbas Khan ${ }^{1}$ \\ ${ }^{1}$ Department of Chemistry, Abdul Wali Khan University Mardan, Mardan, KP 23200, Pakistan \\ ${ }^{2}$ Department of Biochemistry, Shaheed Benazir Bhutto Women University, Peshawar, KP 25000, Pakistan
}

\begin{abstract}
The study and application of reversible addition-fragmentation chain transfer (RAFT) polymerization have been widely reported in the literature because of its high compatibility with numerous monomers, reaction conditions, and low polydispersity index. The effect of RAFT agents on the characteristics of the final product is greatly needed to be explored. Our present study aimed to compare the influence of two different types of RAFT agents on the characteristics of the water-soluble polymer (2acrylamido-2-methylpropane sulfonic acid) (polyAMPS) and their polyAMPS@butyl methacrylate (BMA) core-shell particles. Different analytical techniques including scanning electron microscopy (SEM), fourier transform infrared spectroscopy (FTIR), energy dispersive X-ray analysis (EDX), X-ray diffraction (XRD), and thermogravimetric analysis (TGA) were used to ascertain the final morphological, structural, and thermal properties of the resultant products. It was found that RAFT agents have shown a clear influence on the final properties of the resultant polyAMPS and their core-shell particles such as particle size, shape, size distribution, and thermal behavior. This study confirms that RAFT agents can control the final properties of the polymers and their core-shell particles.
\end{abstract}

Keywords: RAFT agents, effect of RAFT agents, polyAMPS, core-shell particles, characterization

\section{Introduction}

Polymers became the center of research over the past few decades due to their good adaptability $[1,2]$ and various potential applications in the field of medical [3, 4], pharmaceutical [5], and other industrial applications [6,7]. The prerequisite for complete control over the application of polymers is the "polymerization process". The advantages and shortcomings of polymerization process are dependent on its low versatility or compatibility over different monomer, solvent system, provided conditions and suitable initiators [8]. One of the most prominent polymerizations include controlled/living radical polymerization (CLRP). Among all other CLRP, the most advance type of polymerization which uses thiocarbonylthio functionality, provides relatively high versatility over a provided condition (solvent, temperature, $\mathrm{pH}$ and initiator), functional and nonfunctional monomers to yield desire material with com-

*E-mail: nasrchem@gmail.com plex architectures, narrow molecular weight distributions, and pre-determined molecular weights [9]. This thiocarbonylthio functionality is provided by reversible addition-fragmentation chain-transfer (RAFT) agents, which leads to RAFT polymerization. Since the invention of RAFT polymerization, numerous RAFT agents have been synthesized and reported [10].

RAFT polymerizations have been used in the synthesis of various polymeric architectures, such as star [11], brush [11], linear [12], dendrimer [13], core-shell [14], and graft [15], along with different conditions, namely, solution, suspension, emulsion, and miniemulsion polymerizations [16]. Dispersity is one of the prominent parameters that affects the properties of polymers. Control over dispersity can be attained by mixing two RAFT agents with adequately dissimilar chain-transfer behaviors in different ratios, affording polymers with monomodal molecular weight distributions over a broad dispersity range [17]. Henkel and Vana [18] studied the effect of RAFT agents on the mechanical and the microstructure behavior of poly(butyl 
acrylate). For this purpose, a photoinitiated polymerization of 1,4-butanediol diacrylate and butyl acrylate was conducted in the absence and presence of RAFT agents. It was found that RAFTbased polymers have lower Young's moduli and high swelling degree. Moreover, kinetic differential scanning calorimetry studies illustrated that the gel point was retarded with enlarging the content of RAFT agent [18]. Masuda and Takai [19] studied the effect of RAFT agent content on the microstructure and properties of poly( $\mathrm{N}$-isopropylacrylamide) (PNIPAAm) gels. A millimeter-sized cylinder was synthesized from PNIPAAm gels. Swelling and deswelling behaviors were studied, and we found that a cylinder with high "RAFT agent content" showed fast deswelling properties [19]. The various multi-arm RAFT agents have been used in stereolithographic 3D printing. Further, it was widely found that changing the functionality and content of RAFT agents result in obtaining control over material mechanical behavior in a broad span [20]. Application of RAFT polymerization instead of free radical polymerization produced major variations in the mechanical, uptake behaviors and thermal properties, which seem to reflect the improvement in polymer uniformity and mobility frequently related with controlled polymerization [21].

The application and properties of core-shell nanomaterials can be promisingly controlled by the right selection of shell or core materials according to the environment/condition and applications. A vast study of core-shell material "as sensing" device have been reported, i.e., as optical sensors, gas adsorptive sensors, electrochemical sensors, and wearable sensing devices. These devices have various potential uses in food analysis and biological, industrial, environmental, and clinical applications. Moreover, numerous synthetic approaches with various prominent properties of core-shell materials, such as high ion transport properties, high conductivities, and high surface area have been studied. [22]

Although the synthesis of RAFT agents and their use in the preparation has already been reported [23, 24], to our knowledge, their effect on the final characteristics of the prepared water- soluble polymer (2-acrylamido-2-methylpropane sulfonic acid) (polyAMPS) and their core-shell particles has not been reported. Hence, considering various advantages of RAFT polymerization, this effort was made to determine their role in controlling the thermal stability, particle size distribution, crystallinity, and average particle size of the resultant core-shell particles with polyAMPS as a shell and butyl methacrylate (BMA) as a core.

\section{Experimental section}

\subsection{Materials}

Potassium persulfate, sodium hydride, magnesium turnings, and carbon disulfide were purchased from Daejung, Korea. 4-vinylbenzyl chloride, 4,4'-azobis(4-cyanovaleric acid) (ABCA), dimethylformamide (DMF), BMA, 2-acrylamido-2-methylpropane sulfonic acid (AMPS), iodine, magnesium sulfate, potassium persulfate $\left(\mathrm{K}_{2} \mathrm{~S}_{2} \mathrm{O}_{8}\right)$, n-hexane, diethyl ether, bromobenzene, dimethyl sulfoxide, petroleum ether, and tetra hydrofuran were purchased from Sigma-Aldrich. Pyrroles and silica gel were the products of Unichem, USA.

\subsection{Instruments used}

The FTIR analysis was done via Thermo Fisher Scientific model NICOLET iS5. Scanning electron microscopic and energy dispersive X-ray analyses were done via field-emission scanning electron microscopy (SEM), JEOL Japan, model JSM5910, with an acceleration voltage of $30 \mathrm{KV}$. X-ray diffraction (XRD) pattern was recorded via an X-ray diffractometer (model JDX-3532), JEOL Japan, by using Ni-filtered $\mathrm{Cu} \mathrm{K} \alpha$ radiation and a wavelength of $1.5418 \AA$. Thermogravimetric analysis was done with TGA instrument from PerkinElmer (USA), model TGA 4000.

\subsection{Preparation of RAFT agents}

In the study, two different RAFT agents 4-vinylbenzyl pyrrolecarbodithioate and 4vinylbenzyl dithiobenzoate (4VP and 4VD, respectively) were separately prepared using the reported protocols with some modifications [24]. 
Briefly, for the preparation of $4 \mathrm{VP}, 6.02 \mathrm{~g}$ of $\mathrm{NaH}$ was mixed in $160.00 \mathrm{ml}$ of DMF, followed by stepwise addition of $10.02 \mathrm{~g} / 20 \mathrm{ml}$ of pyrrole, 9.01 $\mathrm{ml} / 20 \mathrm{ml} \mathrm{CS} 2$, and $22.2 / 20 \mathrm{ml}$ of 4-vinylbenzyl chloride, which were all dissolved in DMF. The mixture was stirred for $12 \mathrm{~h}$. For the isolation of $4 \mathrm{VP}$, the resultant product was washed with diethyl ether and distilled water (1:1), followed by extraction via column chromatography, in which petroleum ether was used as a mobile phase. The petroleum ether was separated through vacuum distillation, and the final product, 4VP, was stored at $-18{ }^{\circ} \mathrm{C}$ in an inert environment. For the synthesis of $4 \mathrm{VD}, 3.301 \mathrm{~g}$ of magnesium turning was dissolved in $14 \mathrm{ml} / 40 \mathrm{ml}$ bromobenzene (in tetrahydrofuran), and to initiate this reaction, 0.1 $\mathrm{g}$ of iodine was added. $\mathrm{CS}_{2}[7.91 \mathrm{ml} / 5 \mathrm{~mL}$ (in tetrahydrofuran)] and 4-vinylbenzyl chloride [7.19 $\mathrm{mL} / 5 \mathrm{ml}$ (in tetrahydrofuran)] were added in a stepwise manner after stirring for $12 \mathrm{~h}$. The same isolation process was followed for 4VD. Structures of these two RAFT agents are given in Figure 1.

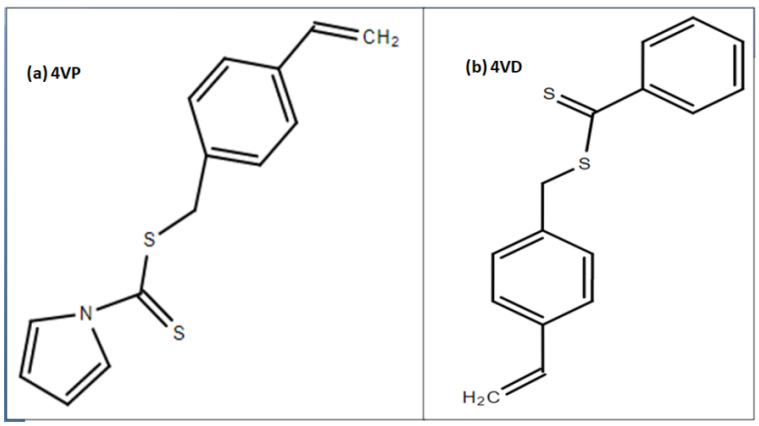

Fig. 1. Structures of the as-prepared RAFT agents. RAFT, reversible addition-fragmentation chaintransfer.

\subsection{Preparation of polyAMPSi and polyAMPSii}

For the preparation of water-soluble polymer poly(2-acrylamido-2-methylpropane sulfonic acid) (polyAMPSi), the previously reported method was adopted with certain minor modifications [23]. Briefly, for the synthesis of polyAMPS $i$, a known amount of AMPS monomer, 4VP (25:1), and ABCA were added to $25 \mathrm{ml}$ of dimethyl sulfox- ide under an oxygen-free environment. The reacting flask was deoxygenated with nitrogen gas purging and vacuum pump followed by fitting it to water bath for $12 \mathrm{~h}$ at $333 \mathrm{~K}$. After $12 \mathrm{~h}$ at $333 \mathrm{~K}$, a brownish color polyAMPS $i$ was prepared which was stored at $268 \mathrm{~K}$ in an inert environment. The same protocol was used for polyAMPSii but 4VP was replaced with 4VD.

\subsection{Preparation of core-shell particles}

For the preparation of CSi, $25 \mathrm{ml}$ of BMA was slowly added to $1 \mathrm{~g} / 300 \mathrm{ml}$ of polyAMPS $i$ solution in distilled water, followed by addition of potassium persulfate $\left(\mathrm{K}_{2} \mathrm{~S}_{2} \mathrm{O}_{8}\right)$ solution $(1 \mathrm{~g} / 15 \mathrm{ml})$. The whole setup was oxygen-free; this was ensured through the usage of an overhead condenser and magnetic stirrer, which was later fitted in a water bath for $12 \mathrm{~h}$ at $333 \mathrm{~K}$. The same protocol was used for preparation of CSii but polyAMPS $i$ was replaced with polyAMPSii.

\subsection{Characterizations}

FTIR analysis was carried out to confirm the presence of various functional groups. TGA analysis was used to study the control of RAFT agents over thermal properties (stability). XRD was done to determine the effect on the semicrystalline nature of the obtained CS particles. The SEM study was used to study the morphology and influence of RAFT agents on the particle size of the obtained CS particles.

\section{Results and discussion}

\subsection{FTIR analysis of polyAMPS and core- shell particles}

The FTIR analysis given in Figure 2 reveals the successful preparation of polyAMPS $i$ and polyAMPS $i i$, which is confirmed by the presence of various characteristic peaks, such as asymmetric and symmetric vibrations of $-\mathrm{SO}_{2}$ at $1,031 \mathrm{~cm}^{-1}$ and $1,210 \mathrm{~cm}^{-1}, \mathrm{~N}-\mathrm{H}$ and $\mathrm{C}=\mathrm{O}$ vibrations at 1,538 $\mathrm{cm}^{-1}$ and $1,665 \mathrm{~cm}^{-1}$, and $-\mathrm{CH}_{3}$ and $-\mathrm{CH}_{2}$ asymmetric and symmetric stretching at 2,996 and 2,913 $\mathrm{cm}^{-1}$, respectively. These peaks are found in the 
structure of polyAMPS, and the data are in agreement with the literature $[25,26]$.

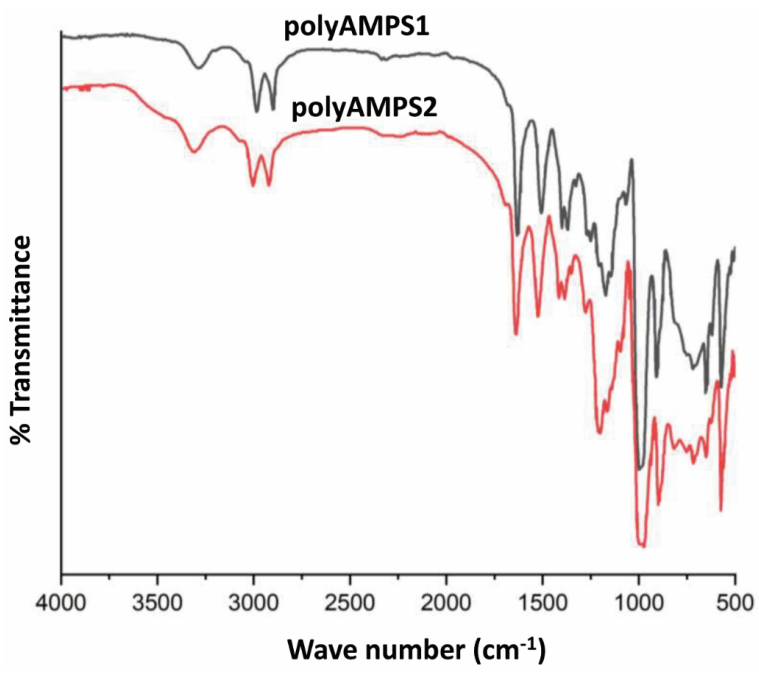

Fig. 2. FTIR spectra of the as-prepared polyAMPS $i$ and polyAMPSii. polyAMPS, poly(2-acrylamido-2methylpropane sulfonic acid).

The CSi and CSii were synthesized with the application of as-prepared polyAMPSi and polyAMPSii. FTIR was carried out to evaluate their structure, and different characteristic peaks have been obtained, which are listed in Table 1 along with their specific regions and corresponding species. Figure 3 represents the two spectra of CSi and CSii. No major differences have been found in these graphs, which confirms that RAFT agents have no affect or influence on the core-shell structure.

The wave numbers axis in the FTIR spectrum of synthesized CSi and CSii is given in Figure 3, which indicates the presence of various functional groups due to the presence of their corresponding characteristic peaks. A peak present at 1,586 $\mathrm{cm}^{-1}$ illustrates the bending vibration of $\mathrm{N}-\mathrm{H}$, while peaks at $1,190 \mathrm{~cm}^{-1}$ and $1,039 \mathrm{~cm}^{-1}$ correspond to symmetric and asymmetric stretching of $-\mathrm{SO}_{2}$; moreover, other peaks at $1,633 \mathrm{~cm}^{-1}, 1,364 \mathrm{~cm}^{-1}$, and $603 \mathrm{~cm}^{-1}$ illustrate the existence of $\mathrm{C}=\mathrm{O}, \mathrm{C}-\mathrm{N}$, and $\mathrm{C}-\mathrm{S}$, respectively. All these functional groups confirmed the existence of polyAMPS in CSi and CSii. Other peaks at $2,957 \mathrm{~cm}^{-1}, 1,726 \mathrm{~cm}^{-1}$, and $1,240 \mathrm{~cm}^{-1}$ correspond to stretching vibration of

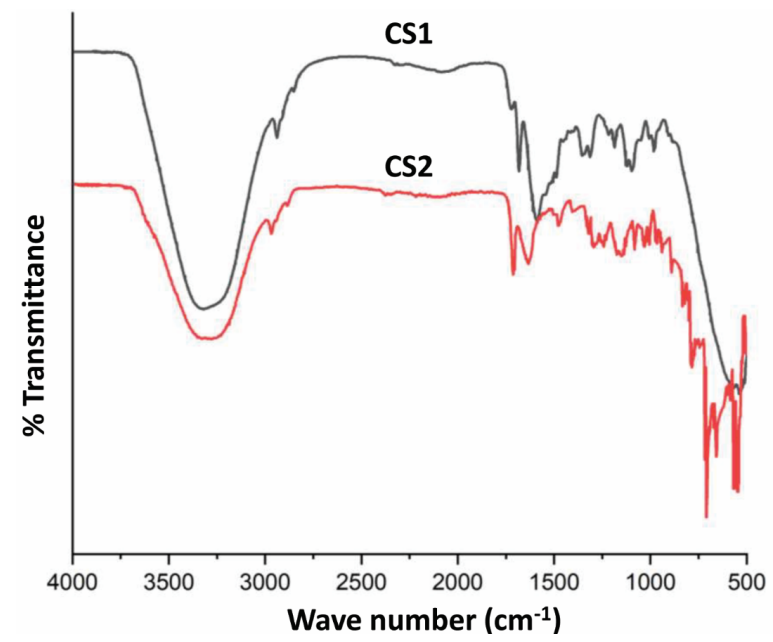

Fig. 3. FTIR spectra of the CS particles.

functional groups of BMA, which are $\mathrm{C}-\mathrm{H}, \mathrm{C}-\mathrm{O}-$ $\mathrm{C}$, and $\mathrm{C}=\mathrm{O}$, respectively. Functional groups with their respective peak regions are listed in Table 1.

\subsection{Thermogravimetric analysis of polyAMPS and core-shell particles}

For studying the influence of RAFT agents on thermal degradation of their respective synthesized polymer, both polyAMPS $i$ and polyAMPS $i i$ were analyzed via thermogravimetry, and a temperature increase rate of $30{ }^{\circ} \mathrm{C} / \mathrm{min}$ was adjusted. The obtained thermogram gave two major degradations: (a) the one between $125{ }^{\circ} \mathrm{C}$ and $200{ }^{\circ} \mathrm{C}$ illustrates thermal degradation of sulfonic acid moiety $[26,28]$ (b) and the second one at 200-300 ${ }^{\circ} \mathrm{C}$ corresponds to polyAMPS chain burning. Initially, there is not much difference in the degradation rates of the two RAFT agents, but it appears at higher temperatures in later stages of thermogravimetric analysis. The degradation rate and extent in polyAMPSii are higher than those in polyAMPSi. A clear difference in thermal stability appears in the prepared polyAMPS after 230 ${ }^{\circ} \mathrm{C}$, and it was observed that polyAMPSii lost its $70 \%$ weight at $243{ }^{\circ} \mathrm{C}$ compared to at $308{ }^{\circ} \mathrm{C}$ for polyAMPSi. After $300^{\circ} \mathrm{C}$, the thermal degradation is very small and the weight of the polyAMPS $i$ and polyAMPSii remains stable. The thermogram given in Figure 4 and Table 2 shows relative high stability of polyAMPSi, which is surely due to the 
Table 1. Identifying characteristic peaks of CSi and CSii particles.

\begin{tabular}{|c|c|c|c|c|}
\hline $\begin{array}{l}\text { S. } \\
\text { No. }\end{array}$ & Wave number $\left(\mathrm{cm}^{-1}\right)$ & Functional group & $\begin{array}{l}\text { Belonging } \\
\text { molecule }\end{array}$ & References \\
\hline 1. & 1,633 & $\mathrm{C}=\mathrm{O}$ & & \\
\hline 2. & 1,583 & $\mathrm{~N}-\mathrm{H}$ (bending) & & \\
\hline 3. & 1,364 & $\mathrm{C}-\mathrm{N}$ & polyAMPS & [25] \\
\hline 4. & 1,190 and 1,039 & $\mathrm{O}=\mathrm{S}=\mathrm{O}$ (asymmetric and symmetric stretching) & & \\
\hline 5. & 603 & $\mathrm{C}-\mathrm{S}$ stretching & & \\
\hline 6. & 3,333 & Vibration of bonded and non-bonded $\mathrm{OH}$ groups & $\mathrm{H}_{2} \mathrm{O}$ & Disperse medium \\
\hline 7. & 2,957 & Stretching $\mathrm{CH}$ & & \\
\hline 8. & 1,726 & $\mathrm{C}=\mathrm{O}$ stretching & BMA & [27] \\
\hline 9. & 1,240 & $\mathrm{C}-\mathrm{O}-\mathrm{C}$ stretching & & \\
\hline
\end{tabular}

BMA, butyl methacrylate; polyAMPS, poly(2-acrylamido-2-methylpropane sulfonic acid).

application of pyrrole-based RAFT agents, as reported by Stace et al. [29], as the dithiocarbamatebased RAFT agents are thermally more stable than other RAFT agents, which is basically due to presence of pyrrole and bidentate legends $(\mathrm{SC}(=\mathrm{S}) \mathrm{N})$ [30]. These factors increase crystallinity along with thermal stability $[29,30]$.

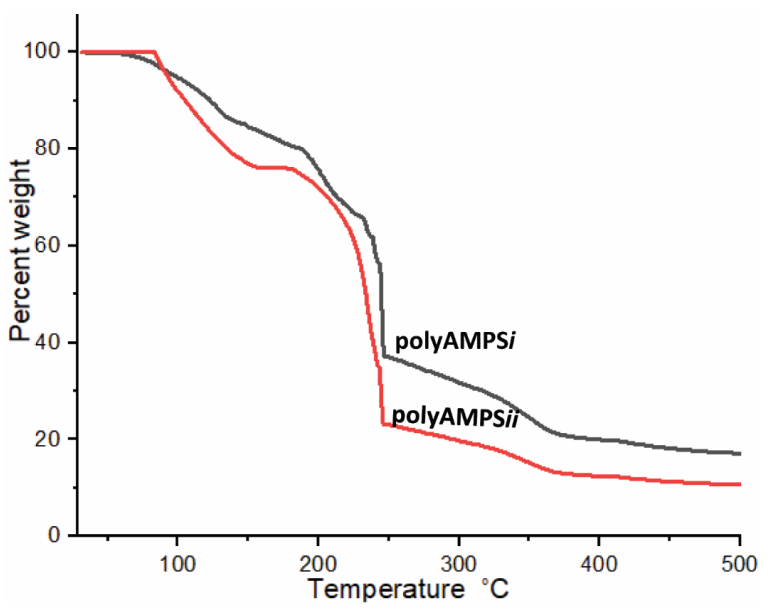

Fig. 4. Comparing TGA results of polyAMPS. polyAMPS, poly(2-acrylamido-2methylpropane sulfonic acid).

Due to relatively high thermal stability of polyAMPS $i$, the corresponding $\mathrm{CS} i$ also showed high thermal stability, which is also elaborated by Figure 5 and Table 3. The thermogram showed three key weight decreases: the first one at 40$190{ }^{\circ} \mathrm{C}$ corresponds to complete dehydration [28];
Table 2. Comparative analysis of thermal degradation with respect to temperature.

\begin{tabular}{llll}
\hline \multirow{2}{*}{ Polymer } & \multicolumn{3}{l}{ Temperature $\left({ }^{\circ} \mathrm{C}\right)$ with weight reduction } \\
\cline { 2 - 4 } & $20 \%$ & $50 \%$ & $70 \%$ \\
\hline \hline polyAMPS $i$ & 177 & 242 & 308 \\
polyAMPS $i i$ & 131 & 232 & 243 \\
\hline
\end{tabular}

polyAMPS, poly(2-acrylamido-2-methylpropane sulfonic acid).

the second degradation occurs in the range of 190$290{ }^{\circ} \mathrm{C}$, which is specific for sulfonic acid groups in polyAMPS which produce sulfur oxide gases $\left(\mathrm{SO}_{2}\right.$ and $\left.\mathrm{SO}_{3}\right)[26,31]$; and the third degradation was between $300{ }^{\circ} \mathrm{C}$ and $450{ }^{\circ} \mathrm{C}$, which shows increased weight degradation of $76.757 \%$ for CSi and $76.269 \%$ for CSii. This major weight lost was due to deesterification [32], depolymerization, and denaturing of BMA [33], in which blazing of a hydrocarbon chain occurred and turned out into carbon monoxide, carbon dioxide, and traces of water vapor $[34,35]$. Another major factor that enhances thermal stability of CSi was its relative high degree of crystallinity [36] since $\mathrm{SC}(=\mathrm{S}) \mathrm{N}$ (present in $4 \mathrm{VP}$ ) has the capacity of being a bidentate legend [30]. A relative more pronounced XRD peak (Figure 6) supports this evidence. 


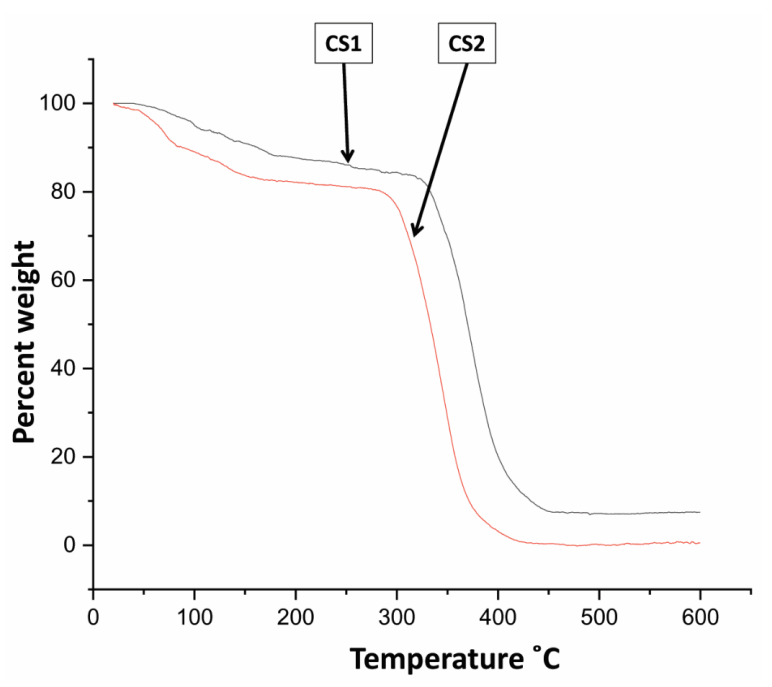

Fig. 5. Comparative TGA results of CS particles.

Table 3. Comparative analysis of thermal degradation with respect to temperature.

\begin{tabular}{|c|c|c|c|c|}
\hline \multirow{2}{*}{$\begin{array}{l}\text { Core-shell } \\
\text { particles }\end{array}$} & \multicolumn{4}{|c|}{ Temperature $\left({ }^{\circ} \mathrm{C}\right)$ with weight reduction } \\
\hline & $25 \%$ & $50 \%$ & $75 \%$ & $92 \%$ \\
\hline $\mathrm{CS} i$ & 340 & 368 & 392 & 446 \\
\hline CSii & 302 & 333 & 352 & 374 \\
\hline
\end{tabular}

\subsection{XRD analysis of the core-shell parti- cles}

The XRD study revealed three major peaks: the first one was a more pronounced peak at $2 \theta=$ $18.82^{\circ}$, corresponding to a high degree of amorphous nature of both core-shell polymeric particles [37, 38], and the second and third peaks at $2 \theta=37^{\circ}$ and $45^{\circ}$ reflect a very small amount of crystallinity. The relative study revealed high crystallinity in CSi with respect to CSii, which is due to the presence of bidentate legends $(\mathrm{SC}(=\mathrm{S}) \mathrm{N})$ in CSii [30].

\subsection{EDX analysis of core-shell particles}

In the EDX study, both core-shell polymeric particles confirmed the presence of all the expected elements with the abundance trend of $\mathrm{C}>\mathrm{O}>\mathrm{S}>\mathrm{K}$. The atomic and weight percentages with their corresponding species are illustrated in Figure 7. The comparative study revealed the resultant relative high carbon content in $\mathrm{CS} i$, which is due to three

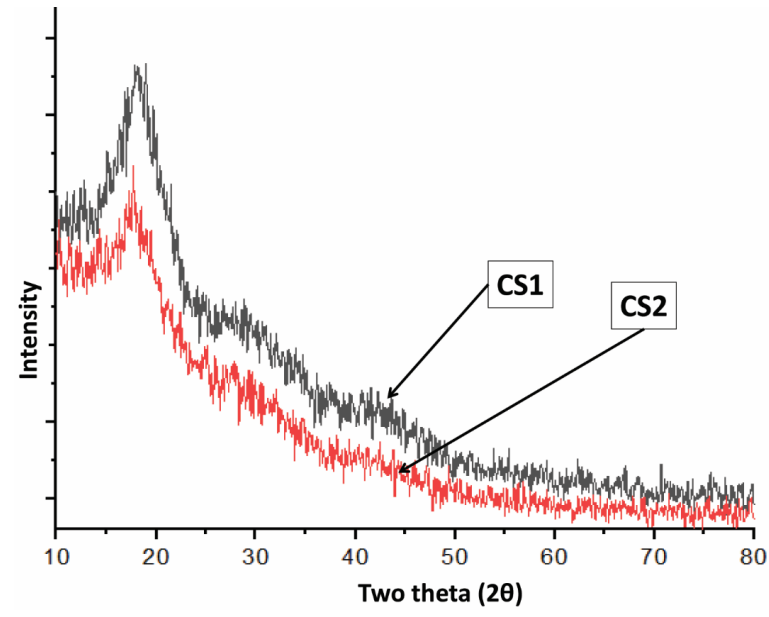

Fig. 6. Comparing XRD results of polymeric CS particles. XRD, X-ray diffraction.

main reasons: high degree of crystallinity, branching, and monomer conversion capacity in CSi [24].

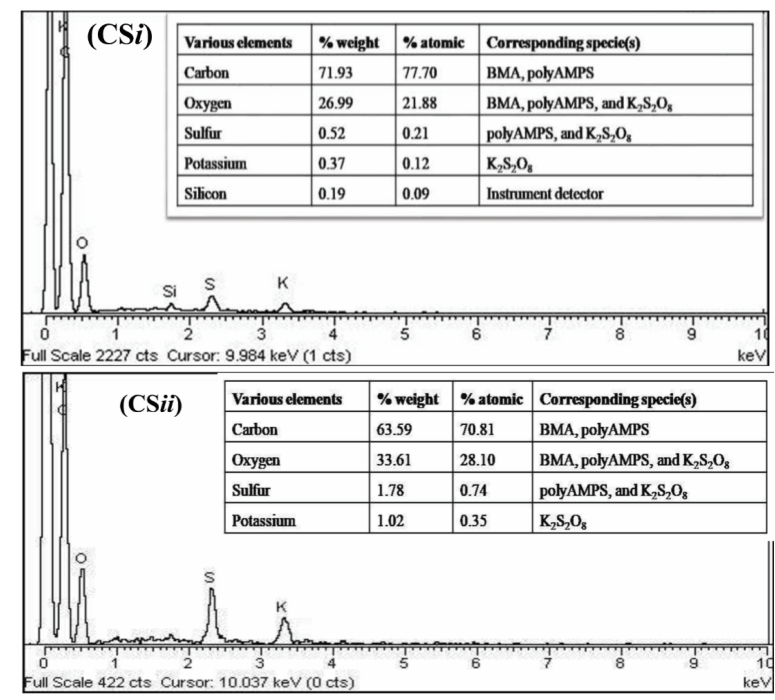

Fig. 7. EDX result of polymeric core-shell particles.

\subsection{SEM analysis}

\subsubsection{Analyzing article shape}

The microscopic analysis of the resultant polyAMPS@BMA core-shell particles was nonuniform. Through SEM study a small portion of crystallinity and large amorphous nature with spherical particles shapes have been observed. Three different particle shapes have been ob- 
tained: spherical, spheroidal, and agglomerated. The shapes of the crystals are needle-like and block-like, with a diameter range from $\sim 10 \mathrm{~nm}$ to $300 \mathrm{~nm}$, which is due to crosslinking [39]. This irregular shape of resultant polyAMPS@BMA coreshell particles provided the advantage of high surface area for various purposes. The relative analysis concluded a high agglomeration in CSii. Particle size distributions (PSDs) are discussed in the following section.
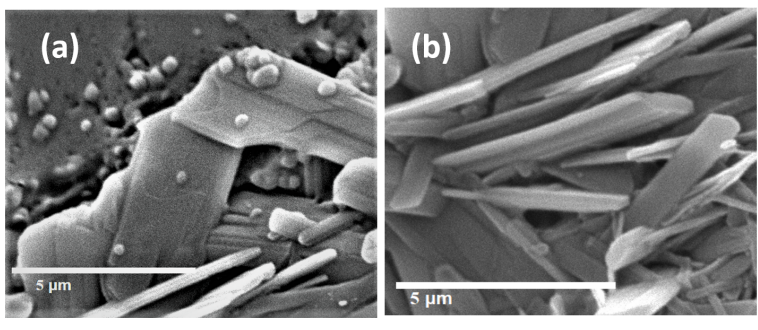

Fig. 8. SEM images (highlighting spherical particles and crystals) of CSi. SEM, scanning electron microscopy.

\subsubsection{Analyzing PSD and core-shell morphol- ogy}

Image J (Java-based image processing program developed at the NIH, USA) software was used to analyze PSD and average particles size. From Figure 10 (left), it can be observed that the sizes of particles for CSi are between $200 \mathrm{~nm}$ and $500 \mathrm{~nm}$, with an average size of $316 \mathrm{~nm}$. Relative maximum particles have sizes ranging from 300 to $350 \mathrm{~nm}$, followed by $250-300 \mathrm{~nm}$. Relatively small amounts have sizes between $450 \mathrm{~nm}$ and $500 \mathrm{~nm}$. The same software was used for CSii, for which particle sizes were distributed between $200 \mathrm{~nm}$ and $600 \mathrm{~nm}$ as illustrated in Figure 10 (right). Relative high numbers of particles were between $350 \mathrm{~nm}$ and $400 \mathrm{~nm}$, with an average particle size of $392 \mathrm{~nm}$. The reason behind average small particle sizes of CSi was the application of $4 \mathrm{VP}$, since it has the capacity of hyper-branching, which results in smaller particle sizes. [24]

Figure 11 shows the scanning electron micrograph images of both core-shell particles. Since SEM analysis was done in bulk form without treatment with any chemical species, a prominent num-

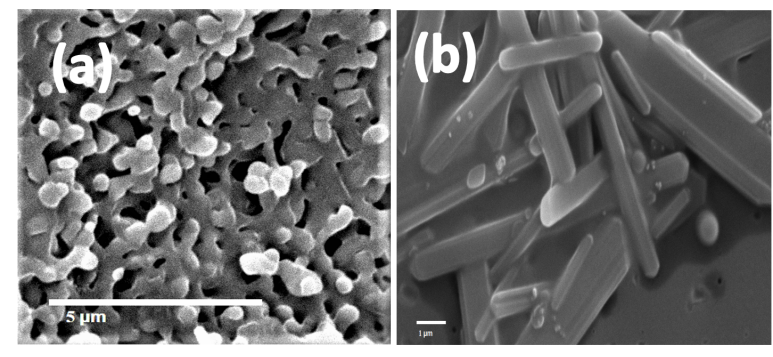

Fig. 9. SEM images (highlighting spherical particles and crystals) of CSii. SEM, scanning electron microscopy.

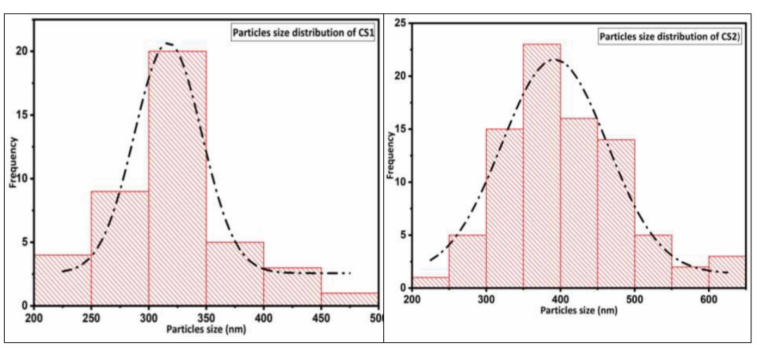

Fig. 10. Histograms of PSD of polymeric CS particles, where the histogram for CSi is on the left and for CSii is on the right. PSD, particle size distribution.

ber of particles were found in agglomerated foam. Non-agglomerated and spherical particles are highlighted with white arrows. Core-shell morphologies have also been confirmed for SEM images. The white blurred portion of each spherical particle illustrates the shell, while the gray inner portion is the core of particles.
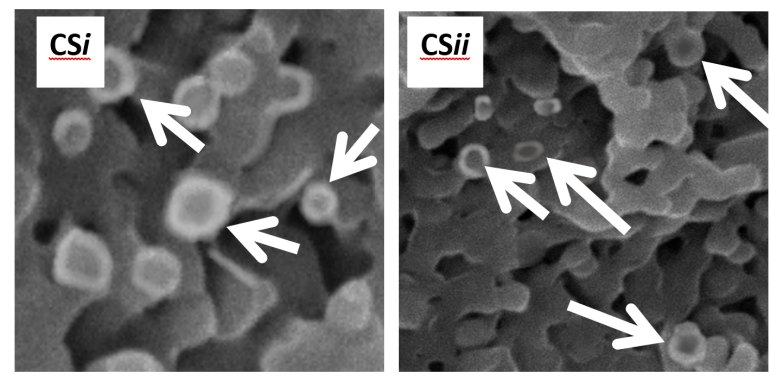

Fig. 11. Core-shell morphologies of obtained particles. 


\section{Conclusion}

The basic purpose of this study was to evaluate the effect of RAFT agents on the net characteristics of the polyAMPS and their resultant coreshell particles. FTIR, XRD, EDX, and SEM studies confirmed the successful synthesis of the RAFT agents, polyAMPS, and polyAMPS@BMA particles. FTIR, XRD, and EDX studies authenticate that with the changing of RAFT agents, the structural properties of the resultant core-shell particles are less affected. The thermal properties, particle sizes, and yields are prominently influenced. Overall, it has been concluded that hyper-branched ability and crystallinity are the major factors that make the RAFT agents to influence the particle size, shape, size distribution, and thermal behavior of obtained polymers.

\section{Acknowledgment}

This work was fully funded by the Higher Education Commission (HEC) of Pakistan under the NRPU Funded Project (5201) awarded to Dr. Nasrullah Shah.

\section{References}

[1] Yang H, Qi D, Liu Z, Chandran BK, Wang T, Yu J, et al. Soft thermal sensor with mechanical adaptability. Adv Mater. 2016;28(41):9175-81.

[2] Kozlovskaya V, Xue B, Kharlampieva E. shapeadaptable polymeric particles for controlled delivery. Macromolecules 2016:49(22):8373-8386.

[3] Feldman D. Polymer nanocomposites in medicine. J Macromol Sci. 2016;53(1):55-62.

[4] Bag MA, Valenzuela LM. Impact of the hydration states of polymers on their hemocompatibility for medical applications: A review. Int J Mol Sci. 2017;18(8):1422.

[5] Parhi R. Cross-linked hydrogel for pharmaceutical applications: A review. Adv Pharm Bull. 2017;7(4):51530.

[6] Hamed I, Özogul F, Regenstein JM. Industrial applications of crustacean by-products (chitin, chitosan, and chitooligosaccharides): A review. Trends Food Sci Technol. 2016;48:40-50.

[7] Wang X. Review of characterization methods for watersoluble polymers used in oil sand and heavy oil industrial applications. Environ Rev. 2016;24(4):460-70.

[8] Matyjaszewski K, Spanswick J. Controlled/living radical polymerization. Mater Today. 20051;8(3):26-33.

[9] Moad G. A critical survey of dithiocarbamate reversible addition-fragmentation chain transfer (RAFT) agents in radical polymerization. J Polym Sci Part A. 2019;57(3):216-27.

[10] Keddie DJ, Moad G, Rizzardo E, Thang SH. RAFT agent design and synthesis. Macromolecules. 2012;45(13):5321-42.

[11] Barner-Kowollik C, Davis TP, Stenzel MH. Synthesis of star polymers using RAFT polymerization: What is possible? Aust J Chem, 2006;59(10):719-27.

[12] Lewis RW, Malic N, Saito K, Evans RA, Cameron NR. Ultra-high molecular weight linear coordination polymers with terpyridine ligands. Chem Sci, 2019;10(24):6174-83.

[13] Caminade AM, Beraa A, Laurent R, Delavaux-Nicot B, Hajjaji M. Dendrimers and hyper-branched polymers interacting with clays: Fruitful associations for functional materials. J Mater Chem A, 2019;7(34):19634-50.

[14] Pourjavadi A, Rahemipoor S, Kohestanian M. Synthesis and characterization of multi stimuli-responsive block copolymer-silica hybrid nanocomposites with core-shell structure via RAFT polymerization. Compos Sci Technol, 2020;188:107951.

[15] Zhu Y, Egap E. PET-RAFT polymerization catalyzed by cadmium selenide quantum dots (QDs): Grafting-from QDs photocatalysts to make polymer nanocomposites. Polym Chem, 2020;11(5):1018-24.

[16] György C, Lovett JR, Penfold NJ, Armes SP. Epoxy-functional sterically stabilized diblock copolymer nanoparticles via RAFT aqueous emulsion polymerization: Comparison of two synthetic strategies. Macromol Rapid Commun, 2019;40(2):1800289.

[17] Whitfield R, Parkatzidis K, Truong NP, Junkers $\mathrm{T}$, Anastasaki A. Tailoring polymer dispersity by RAFT polymerization: A versatile approach. Chem, 2020;6(6):1340-52.

[18] Henkel R, Vana P. The influence of RAFT on the microstructure and the mechanical properties of photopolymerized poly (butylacrylate) networks. Macromol Chem Phys, 2014;215(2):182-9.

[19] Masuda T, Takai M. Structure and properties of thermoresponsive gels formed by RAFT polymerization: Effect of the RAFT agent content. Polym J, 2020;52(12):1407-12.

[20] Shi X, Zhang J, Corrigan N, Boyer C. PET-RAFT facilitated 3D printable resins with multifunctional RAFT agents. Mater Chem Front, 2021;5(5):2271-82.

[21] Benaddi AO, Cohen O, Matyjaszewski K, Silverstein MS. RAFT polymerization within high internal phase emulsions: Porous structures, mechanical behaviors, and uptakes. Polymer, 2021;213:123327.

[22] Kalambate PK, Huang DZ, Li Y, Shen Y, Xie M, Huang Y, et al. Core@ shell nanomaterials based sensing devices: A review. TrAC Trends Anal Chem, 2019;115:147-61.

[23] Platt L, Kelly L, Rimmer S. Controlled delivery of cytokine growth factors mediated by core-shell particles with poly (acrylamidomethylpropane sulphonate) shells. J Mater Chem B, 2014;2(5):494-501. 
[24] Shallcross L, Roche K, Wilcock CJ, Stanton KT, Swift $\mathrm{T}$, Rimmer S, et al. The effect of hyperbranched poly (acrylic acid) s on the morphology and size of precipitated nanoscale (fluor) hydroxyapatite. J Mater Chem B, 2017;5(30):6027-33.

[25] Clara I, Lavanya R, Natchimuthu N. pH and temperature responsive hydrogels of poly (2-acrylamido-2-methyl1-propanesylfonic acid-co-methacrylic acid): Synthesis and swelling characteristics. J Macromol Sci, Part A, 2016;53(8):492-9.

[26] Erkartal M, Aslan A, Erkilic U, Dadi S, Yazaydin $\mathrm{O}$, Usta H, Sen U. Anhydrous proton conducting poly (vinyl alcohol) (PVA)/poly(2-acrylamido2-methylpropane sulfonic acid)(PAMPS)/1, 2, 4triazole composite membrane. Int J Hydrogen Energy, 2016;41(26):11321-30.

[27] Feng Y, Xiao CF. Research on butyl methacrylate-lauryl methacrylate copolymeric fibers for oil absorbency. $\mathrm{J}$ Appl Polym Sci, 2006;101(3):1248-51.

[28] Qiao J, Hamaya T, Okada T. New highly protonconducting membrane poly(vinylpyrrolidone)(PVP) modified poly (vinylalcohol)/2-acrylamido-2-methyl1-propanesulfonic acid (PVA-PAMPS) for low temperature direct methanol fuel cells (DMFCs). Polymer, 2005;46(24):10809-16.

[29] Stace SJ, Fellows CM, Moad G, Keddie DJ. Effect of the Z-and macro R-group on the thermal desulfurization of polymers synthesized with acid/base "Switchable" dithiocarbonate RAFT agents. Macromol Rapid Commun, 2018;39(19):1800228.

[30] Cotton FA, Wilkinson G, Murillo CA, Bochmann M, Grimes R. Advanced inorganic chemistry. Wiley: New York; 1988.

[31] Erkartal M, Usta H, Citir M, Sen U. Proton conducting poly (vinyl alcohol)(PVA)/poly (2-acrylamido-2methylpropane sulfonic acid)(PAMPS)/zeolitic imidazolate framework (ZIF) ternary composite membrane. J Membrane Sci, 2016;499:156-63.

[32] Novakovic K, Katsikas L, Popovic IG. The thermal degradation of poly (iso-butyl methacrylate) and poly (sec-butyl methacrylate). J Serbian Chem Soc (Yugoslavia), 2000;65(12):867-75.

[33] Herrera-Alonso JM, Sedlakova Z, Marand E. Gas barrier properties of nanocomposites based on in situ polymerized poly (n-butyl methacrylate) in the presence of surface modified montmorillonite. J Membrane Sci, 2010;349(1-2):251-7.

[34] Mohamed OA, Moustafa AB, Mehawed MA, El-Sayed NH. Styrene and butyl methacrylate copolymers and their application in leather finishing. J Appl Polym Sci, 2009;111(3):1488-95.

[35] Liao YH, Rao MM, Li WS, Yang LT, Zhu BK, Xu $\mathrm{R}$, et al. Fumed silica-doped poly (butyl methacrylatestyrene)-based gel polymer electrolyte for lithium ion battery. J Membrane Sci, 2010;352(1-2):95-9.

[36] Song F, Wang Q, Wang T. The effects of crystallinity on the mechanical properties and the limiting PV (pressure $\times$ velocity) value of PTFE. Tribol Int, 2016;93:1-0.

[37] Suhailath K, Ramesan MT, Naufal B, Periyat P, Jasna VC, Jayakrishnan P. Synthesis, characterisation and flame, thermal and electrical properties of poly (n-butyl methacrylate)/titanium dioxide nanocomposites. Polym Bull, 2017;74(3):671-88.

[38] Boroujeni KP, Tohidiyan Z, Fadavi A, Eskandari MM, Shahsanaei HA. Synthesis and catalytic application of poly (2-acrylamido-2-methyl-1-propanesulfonic acidco-acrylamide) grafted on graphene oxide. ChemistrySelect, 2019;4(26):7734-44.

[39] Zhang L, Gao H, Liao Y. Preparation and application of poly(AMPS-co-DVB) to remove Rhodamine B from aqueous solutions. React Funct Polym, 2016;104:53-61.

Received 19-05-2021

Accepted 24-06-2021 